\title{
Intrabeam scattering in high brightness electron linacs
}

\author{
S. Di Mitri \\ Elettra-Sincrotrone Trieste, 34149 Basovizza, Trieste, Italy
}

(Received 19 May 2014; published 8 July 2014)

\begin{abstract}
We studied analytically and with particle tracking the intrabeam scattering of a high brightness electron beam in a linac. We found that intrabeam scattering plays no significant role in the microbunching instability that develops in linacs for free electron lasers. A partial damping of the instability is envisaged, however, either with dedicated magnetic insertions, or with electron beams of charge density $\sim 4$ times higher than produced by state-of-the-art photoinjectors.
\end{abstract}

PACS numbers: 29.20.Ej, 29.27.Bd, 41.60.Cr, 41.85.Ja

\section{WORK PLAN}

The question to which extent intrabeam scattering (IBS) affects the properties of high brightness electron bunches in linacs was posed in [1,2], with attention to the interplay of IBS and microbunching instability (MBI). The present study aims to provide a quantitative answer and an outlook, by comparing the analysis and particle tracking runs of the ELEGANT code [3]. IBS was simulated following the prescriptions given in $[2,4]$.

In Sec. II, we estimate the IBS-induced growth rate of an electron bunch's slice transverse emittance and slice energy spread with the formalism introduced by Piwinski $[5,6]$ and reworked in [1]. For completeness, we send the reader to [7-9] for other recent studies on IBS. In this context, "slice" means local in the longitudinal particle coordinate along the bunch. It was demonstrated $[10,11]$ in the LCLS [12] and the FERMI [13] free electron laser (FEL) facility that incoherent energy spread may Landau damp the MBI [14-16]. The instability would otherwise modulate the electron beam longitudinal phase space in the presence of bunch length magnetic compression, with a detrimental impact to the generation of high brilliance radiation from FELs. The energy spread is enlarged from typical values in the range $1-3 \mathrm{keV}$ rms [17] to $5-40 \mathrm{keV}$ rms by a dedicated tool named laser heater (LH) [18], which runs at beam energies around $100 \mathrm{MeV}$. Other techniques [19-21] have been proposed to suppress the MBI, but they have not found an application yet. In Sec. III, we wonder whether IBS could play a role in the same context and propose to shrink the beam transverse dimension with strong focusing ("low-beta") [focusing-defocusing (FODO)] cells, so to increase the IBS longitudinal growth rate.

At first glance, the idea of using IBS to heat an electron bunch traveling in a dedicated FODO channel seems to be

Published by the American Physical Society under the terms of the Creative Commons Attribution 3.0 License. Further distribution of this work must maintain attribution to the author(s) and the published article's title, journal citation, and DOI. attractive for the following reasons: (i) IBS heats the beam by avoiding cost, complexities and maintenance of a $\mathrm{LH}$ system; (ii) the heating level is tunable with the quadrupoles' focusing strength; (iii) it provides longitudinally uncorrelated energy spread, thus avoiding any side effect associated to the energy modulation induced in a $\mathrm{LH}$ at the infrared laser wavelength (e.g., the so-called trickle heating) [22]. We will see however that, to be as effective as a $\mathrm{LH}$, the enhancement of IBS requires a long and densely packed FODO channel, in contrast to the relatively compact layout of a LH. An alternative compact lattice in which the beam recirculates through low-beta FODO channels is investigated in Sec. IV. This solution, however, turns out to be not practical because of the coherent synchrotron radiation (CSR) instability that develops through the arcs. We conclude that a practical enhancement of IBS in a single-pass FODO channel requires charge densities at least $\sim 4$ times higher than those generated by state-of-the-art photoinjectors.

\section{ANALYTICAL MODEL}

\section{A. Background}

Ultrarelativistic electron bunches in modern accelerators generally have much smaller velocity spread in the longitudinal direction of motion than in the transverse planes owing to the relativistic contraction by the Lorentz factor $\gamma$ : $\sigma_{\delta} / \gamma \ll \sigma_{x^{\prime}}, \sigma_{y^{\prime}}$, where $\sigma_{\delta}$ is the beam rms fractional energy spread and $\sigma_{x^{\prime}, y^{\prime}}$ the rms angular divergence. If the bunch's charge density is high enough and the bunch travels a long path, multiple Coulomb scattering tends to redistribute the beam momenta from the transverse degree of freedom to the longitudinal one. This process is called IBS and its longitudinal growth rate may be comparable to the beam damping time in low emittance electron storage rings [23-25]. The instantaneous growth rate of the energy spread of a bunched beam circulating in a ring was given in $[5,6]$. Since there are no synchrotron oscillations in a linac, the formula for a coasting beam should be used here 
(which results in a growth rate a factor 2 larger than that of a bunched beam) [5]:

$$
\frac{1}{\tau_{\delta}} \equiv \frac{1}{\sigma_{\delta}} \frac{d \sigma_{\delta}}{d t}=\frac{2 r_{e}^{2} c N}{64 \pi^{2} \beta^{3} \gamma^{2} \varepsilon_{n, x} \varepsilon_{n, y} \sigma_{z} \sigma_{\delta}} f\left(\frac{\sigma_{\delta}}{\gamma \sigma_{x^{\prime}}}, \frac{\sigma_{\delta}}{\gamma \sigma_{y^{\prime}}}, \sigma_{\delta} \beta \sqrt{\frac{2 \sigma_{y}}{r_{e}}}\right) .
$$

Here $r_{e}$ is the electron classical radius, $\beta c$ the electron velocity, $N$ the number of electrons in the bunch, $\varepsilon_{n, x}, \varepsilon_{n, y}$ the rms normalized transverse emittances and $\sigma_{z}$ the rms bunch length. For a transversely round beam with cylindrical symmetry and $\beta \approx 1$, Eq. (1) reduces to [1]

$$
\frac{1}{\sigma_{\delta}} \frac{d \sigma_{\delta}}{d t} \approx \frac{r_{e}^{2} c N}{8 \gamma^{2} \varepsilon_{n, x} \sigma_{x} \sigma_{z} \sigma_{\delta}^{2}} \ln \left(\frac{\Delta \gamma_{\max }}{\Delta \gamma_{\min }}\right),
$$

where the argument of the Coulomb logarithm is the ratio of the maximum and the minimum energy exchange due to a single scattering event, and $\Delta \gamma_{\max } \propto \gamma^{2} \sigma_{x^{\prime}}, \Delta \gamma_{\min } \propto$ $r_{e} /\left(\sigma_{x} \sigma_{x^{\prime}}\right) \approx \gamma r_{e} / \varepsilon_{n, x}$ [1]. Following an argument made in [26], we consider that the IBS energy distribution has a nearly Gaussian core with a long tail. Since we are mostly interested in the energy spread of the Gaussian core, we set the maximum energy transfer to $\Delta \gamma_{\max }=\gamma \times 10^{-5}$ as also done in [1], and find that the Coulomb logarithm is of the order of 10 for a normalized emittance of the order of $1 \mu \mathrm{m}$. Then, Eq. (2) can be integrated and it yields to the final fractional rms energy spread in the presence of IBS cumulated over the distance $\Delta s$ :

$$
\sigma_{\delta} \approx \sqrt{\sigma_{\delta, 0}^{2}+\frac{2 r_{e}^{2} N}{\gamma^{2} \varepsilon_{n, x} \sigma_{x} \sigma_{z}} \Delta s} \equiv \sqrt{\sigma_{\delta, 0}^{2}+\sigma_{\delta, \mathrm{IBS}}^{2}},
$$

with $\sigma_{\delta, 0}$ the initial rms fractional incoherent energy spread. While the energy spread is increased with the longitudinal rate in Eq. (2), the transverse emittances are decreased at the smaller rate $-\frac{1}{4 \tau_{\delta}}\left(\frac{\sigma_{\delta}}{\gamma \sigma_{x^{\prime}}}\right)^{2}$ [1]. It is worth noticing that Eq. (3) is an approximate expression for smooth betatron oscillations, neither energy dispersion nor particle acceleration. All these assumptions in fact fit the study reported in the remainder of this article.

\section{B. IBS in FEL linac drivers}

In Ref. [1], the author concluded that $\sigma_{\delta, \text { IBS }} \approx 10^{-5}$ cumulated by a $1 \mathrm{nC}$ charge beam at the end of the $14 \mathrm{GeV}, 1 \mathrm{~km}$ long LCLS linac were negligible with respect to the FEL energy-normalized bandwidth $\rho \approx 10^{-4}$ [27], for lasing at the fundamental wavelength $\lambda=0.1 \mathrm{~nm}$. Therefore, IBS would not affect the self-amplified spontaneous emission (SASE) FEL [27-29] performance at LCLS. It is worth noticing that this statement remains valid over a wide range of (lower) beam energies and FEL wavelengths. In fact, if the LCLS beam energy were lowered from 14 to $\sim 1 \mathrm{GeV}$, Eq. (3) would predict an increase of $\sigma_{\delta \text {, IBS }}$ to $\sim 10^{-4}$, and $\rho$ would enlarge up to $\sim 10^{-3}$ because of the longer FEL wavelength resonant at the lower beam energy. A different scenario may occur for externally seeded FELs, which are more sensitive to the slice energy spread than SASE FELs at the same fundamental wavelength $[30,31]$.

If we limit the application of Eq. (3) to the low energy part of a linac, we find that an electron beam from a stateof-the-art photoinjector, e.g., with beam charge $\mathrm{Q}=0.5 \mathrm{nC}$, $\sigma_{z}=750 \mu \mathrm{m}, \varepsilon_{n}=0.6 \mu \mathrm{m} \mathrm{rad}, \sigma_{x}=150 \mu \mathrm{m}$ and $\gamma=300$, collects an absolute rms energy spread $\sigma_{E \text {.IBS }} \approx 3 \mathrm{keV}$ over $\Delta \mathrm{s} \sim 30 \mathrm{~m}$. This is comparable to the typical value of $\sigma_{E, 0} \approx 2 \mathrm{keV}$ out of a photoinjector [17] and still far from the amount of heating required to suppress MBI in an FEL driver $[10,11]$. Then, if we assume that the bunch length is magnetically compressed by a factor of, say, $\mathrm{C} \sim 30, \sigma_{E, \mathrm{IBS}}$ may grow up to $\sim 100 \mathrm{keV}$ over hundreds of meters, but its contribution to Landau damping remains small for two reasons. First, in the linac region immediately following the compressor, $\sigma_{E, \text { IBS }}$ is negligible compared to the incoherent energy spread of the compressed beam, which is increased to $\mathrm{C} \sigma_{E, 0} \approx 60 \mathrm{keV}$ by virtue of the preservation of the longitudinal emittance. Second, $\sigma_{E \text {,IBS }}$ grows with $s$ at a lower rate than the relative energy modulation amplitude of MBI (respectively, square root vs linear dependence) $[5,14]$. In conclusion, the impact of $\sigma_{E, \text { IBS }}$ on the development of the MBI is expected to be small. In particular, it is negligible with respect to the effect of a LH unless important modifications to the magnetic lattice and/or to the beam parameters are introduced. We finally remark that, with the aforementioned beam parameters, the transverse emittances and the bunch length are substantially unchanged by IBS.

\section{LOW-BETA FODO CHANNEL}

\section{A. Analytical estimate}

We asked ourselves if there is any way to increase $1 / \tau_{\delta}$ [see Eqs. (1) and (2)] in the low energy region of an electron linac, to the extent where it can build up several $\mathrm{keV}$ rms energy spread. By using Eq. (3) we found that, for injected beam parameters like those listed in Table I, $\sigma_{E, \text { IBS }} \approx 6 \mathrm{keV}$

TABLE I. Electron beam parameters out of a state-of-the-art photoinjector and FODO lattice parameters.

\begin{tabular}{lcc}
\hline \hline Charge & 500 & $\mathrm{pC}$ \\
Bunch duration, rms & 2.5 & $\mathrm{ps}$ \\
Normalized slice emittance, rms & 0.6 & $\mu \mathrm{m}$ \\
Incoherent energy spread, rms & 2.0 & $\mathrm{keV}$ \\
Mean energy & 150 & $\mathrm{MeV}$ \\
FODO length & 30 & $\mathrm{~m}$ \\
Average betatron function in FODO & 0.3 & $\mathrm{~m}$ \\
IBS-induced energy spread, rms [Eq. (3)] & 6.0 & $\mathrm{keV}$ \\
\hline \hline
\end{tabular}




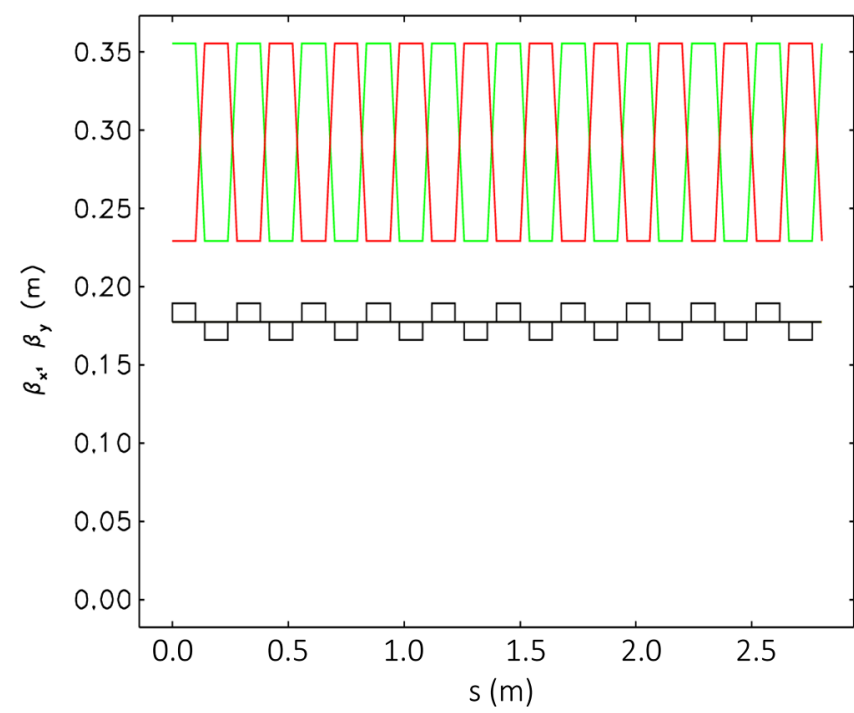

FIG. 1. Horizontal and vertical betatron function in the proposed FODO channel. Beam energy is $150 \mathrm{MeV}$. Quadrupole magnets' field gradient is $50 \mathrm{~T} / \mathrm{m}$ over a magnetic length of $0.1 \mathrm{~m}$. The magnetic field at the quadrupole's bore radius of $10 \mathrm{~mm}$ is $0.5 \mathrm{~T}$. The magnets are separated by $0.04 \mathrm{~m}$.

if the rms transverse beam size $\sigma_{x}$ shrinks down to $25 \mu \mathrm{m}$ (average value) over $30 \mathrm{~m}$. This is done with a FODO channel characterized by a small average betatron function $\bar{\beta}$, as shown in Fig. 1. With such a system designed for the maximum beam heating, i.e., minimum $\bar{\beta}$, a reduction of the total $\sigma_{E, \text { IBS }}$ can be obtained by rearranging the quadrupole strengths so to allow $\bar{\beta}$ to expand to higher values. On the opposite, the lower limit of $\bar{\beta}$ is set by the optical aberrations excited by strong focusing and by the technical design of the quadrupole magnets. State-of-the-art final focusing systems promise a betatron function as small as a

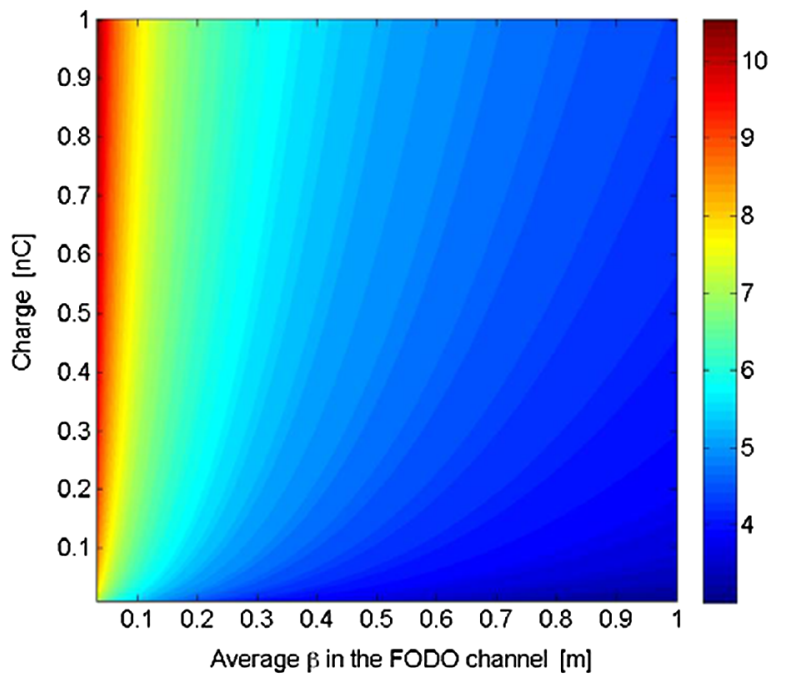

few mm over $\sim \mathrm{mm}$ long drift section [32]. In order to make our system more flexible, compact and easy to build, we set $\bar{\beta}=0.3 \mathrm{~m}$. This solution ensures a standard technical design of the quadrupole magnets and negligible emittance growth in the presence of optical aberrations.

The $\sigma_{E, \text { IBS }}$ cumulated in the FODO channel is evaluated with Eq. (3) and shown in Fig. 2, in the $(\bar{\beta}, \mathrm{Q})$ and the $(\bar{\beta}, \mathrm{L})$ space, with $\mathrm{L}$ the FODO channel total length. We assume that the three-dimensional charge density out of the photoinjector remains constant as the injected bunch charge is varied. In other words we assume the following scaling: $\varepsilon_{n}[\mu \mathrm{m}] \approx Q[\mathrm{nC}]^{1 / 3}$ and $\sigma_{z}[\mathrm{~mm}] \approx 1.2 \times Q[\mathrm{nC}]^{1 / 3}$, so that $Q /\left(\sigma_{z} \varepsilon_{n}^{2}\right)=$ const. This assumption roughly fits experimental data collected both at LCLS and at FERMI, for Q in the range $0.02-1.0 \mathrm{nC}$ [33]. The normalized transverse emittance is here intended to be slice and its value is assumed to be $80 \%$ of the projected value listed in [33]. In general, $\sigma_{E, \text { IBS }}$ turns out to be quite insensitive to $\mathrm{Q}$ if compared to its dependence on $s, \gamma$ and $\bar{\beta}$ because, as shown in Eq. (3), the effect of a higher charge is compensated by a longer bunch duration and a larger transverse emittance.

\section{B. Particle tracking}

We benchmarked the analytical estimation of $\sigma_{\delta, \text { IBS }}$ for the beam parameters in Table I, with particle tracking runs of the ELEGANT code. ELEGANT implements Bjorken and Mtingwa's formulas [34] for calculating the emittance growth rate in all directions of motion. To take into account non-Gaussian distributed beams, ELEGANT allows beam slice analysis: within each slice, particles are assumed to be Gaussian distributed in the transverse phase space and in energy, and uniformly distributed in $z[2,4]$. In the following, we report $\sigma_{\delta \text {,IBS }}$ predicted by ELEGANT for the sliced and the unsliced bunch.



FIG. 2. IBS-induced rms energy spread in $\mathrm{keV}$, in the $(\bar{\beta}, \mathrm{Q})$ space for $\mathrm{L}=30 \mathrm{~m}$ (left), and in the $(\bar{\beta}, \mathrm{L})$ space for $\mathrm{Q}=500 \mathrm{pC}$. Both plots are for a beam energy of $150 \mathrm{MeV}$. The beam transverse emittances and the bunch duration are scaled with $\mathrm{Q}$ as explained in the text above. Notice that the color scale is different in the two plots. 



FIG. 3. Electron beam slice rms fractional energy spread along the FODO channel in the presence of IBS, for the sliced (left) and unsliced beam. Beam energy is $150 \mathrm{MeV}$. For the sliced beam, the fractional energy spread is shown for each slice; its average value over all slices is also shown with the solid line. In the legend, " $\sigma_{\mathrm{d} . I n p u t}$ " is the energy spread at the entrance of each "IBS module" depicted in ELEGANT; " $\sigma_{\mathrm{d}}$ " is the energy spread at the exit of each IBS module and " $\sigma_{\mathrm{d}, \text { Ave }}$ " is the rms fractional energy spread, averaged over all bunch slices. An "IBS module" is a zero-length lattice element; ELEGANT calculates the integrated IBS growth rates between the modules, then scatters particles at each module. The beam's parameters are updated for use in downstream elements. For the unsliced beam, the rms fractional energy spread estimated with Eq. (3) is also shown (circles).


FIG. 4. IBS longitudinal (top) and transverse (bottom) growth rate for sliced (left) and unsliced bunch (right), along the FODO channel. Each point corresponds to one slice. 
The initial current profile is parabolic and the peak current is $65 \mathrm{~A}$. We generated the initial beam with a fractional energy spread $\approx 0.2 \%$ at $150 \mathrm{MeV}$, linearly correlated with the longitudinal coordinate along the bunch (linear energy chirp). Such energy spread is taken into account by the ELEGANT code to compute optical chromatic aberrations. The initial slice energy spread is approximately the total one divided by the number of slices. With 150 slices, the slice energy spread turns out to be $\approx 2 \times 10^{-3} \times 1.5 \times 10^{5} \mathrm{keV} / 150 \cong 2 \mathrm{keV}$, a value consistent with the incoherent energy spread in Table I. Thus, we need to bin the bunched beam in at least 150 slices to investigate the incoherent heating induced by IBS in the presence of the aforementioned energy chirp. We actually chose the conservative value of 300 slices in ELEGANT and 5000 particles per slice. The input charge distribution was generated in ELEGANT and properly smoothed in the six-dimensional phase space to reduce the numerical sampling noise [3].

The incoherent energy spread induced by IBS along the FODO channel is shown in Fig. 3. Its final rms value, averaged over the bunch slices, is $4.5 \mathrm{keV}$ for the sliced beam and $6.0 \mathrm{keV}$ for the unsliced one. Such a discrepancy is due to the nonuniform heating of the sliced beam because of the lower charge density at the bunch edges. The simulations confirm that the bunch length remains substantially unchanged in the presence of IBS (not shown). The IBS transverse and longitudinal growth rate is shown in Fig. 4. In agreement with the low IBS transverse growth rate, the beam transverse emittances change by less than $0.01 \mu \mathrm{m}$ due to momenta transfer. A similarly negligible effect is also from optical aberrations (not shown). By scaling the sliced beam result with Eq. (3), we estimate a FODO channel as long as $\sim 100 \mathrm{~m}$ to achieve $\sigma_{E, \text { IBS }} \sim 10 \mathrm{keV}$. At this point, the scheme would start having a large impact on the machine design and cost. Alternatively, while keeping the $30 \mathrm{~m}$ long FODO channel, a beam charge density $\sim 4$ times higher than in Table I should be provided, which seems to be out of the horizon of present facilities. We can therefore conclude that a relatively compact single-pass low-beta FODO channel could only about double the incoherent energy spread of typical high brightness electron beams produced by nowadays photoinjectors. This is not sufficient for best performance of x-ray FELs, although it might be suitable, e.g., for longer wavelength FELs driven by shorter linacs, lower peak current and/or requiring weaker magnetic compression than in FERMI and LCLS, i.e., having a lower MBI gain.

\section{RECIRCULATING IBS}

As an alternative to the single-pass FODO channel, we investigated a recirculating IBS beam line (RIBS) to cumulate a larger $\sigma_{E, \text { IBS }}$ and minimize the impact on the total linac length. The bunch is injected into and extracted from the RIBS by fast kicker magnets. After $M$ turns into the RIBS, the beam has passed through a low-beta FODO channel $2 \mathrm{M}+1$ times. A sketch of the RIBS at $150 \mathrm{MeV}$ with realistic sizes is shown in Fig. 5. The two arcs are a copy of the design by Douglas et al. [35]. In the present arrangement, the arcs are achromatic and quasiisochronous $\left(\mathrm{R}_{56}=2 \times 10^{-4} \mathrm{~m}, \mathrm{~T}_{566}=4 \times 10^{-3} \mathrm{~m}\right)$ and connected to the FODO channels by matching sections made of additional quadrupole magnets. An ultrarelativistic bunch takes approximately $360 \mathrm{~ns}$ to make one turn in the RIBS. Kickers with rise and fall time pulse duration of a few tens of nanosecond are therefore adequate for our purposes.

Beam dynamics in RIBS was studied with the ELEGANT code, testing several initial beam charges in the range $100-500 \mathrm{pC}$. The initial beam parameters were defined according to the aforementioned scaling rule with beam charge, for the transverse emittance and the bunch length. As an example, we show in Fig. 6(a) $150 \mathrm{MeV}$,

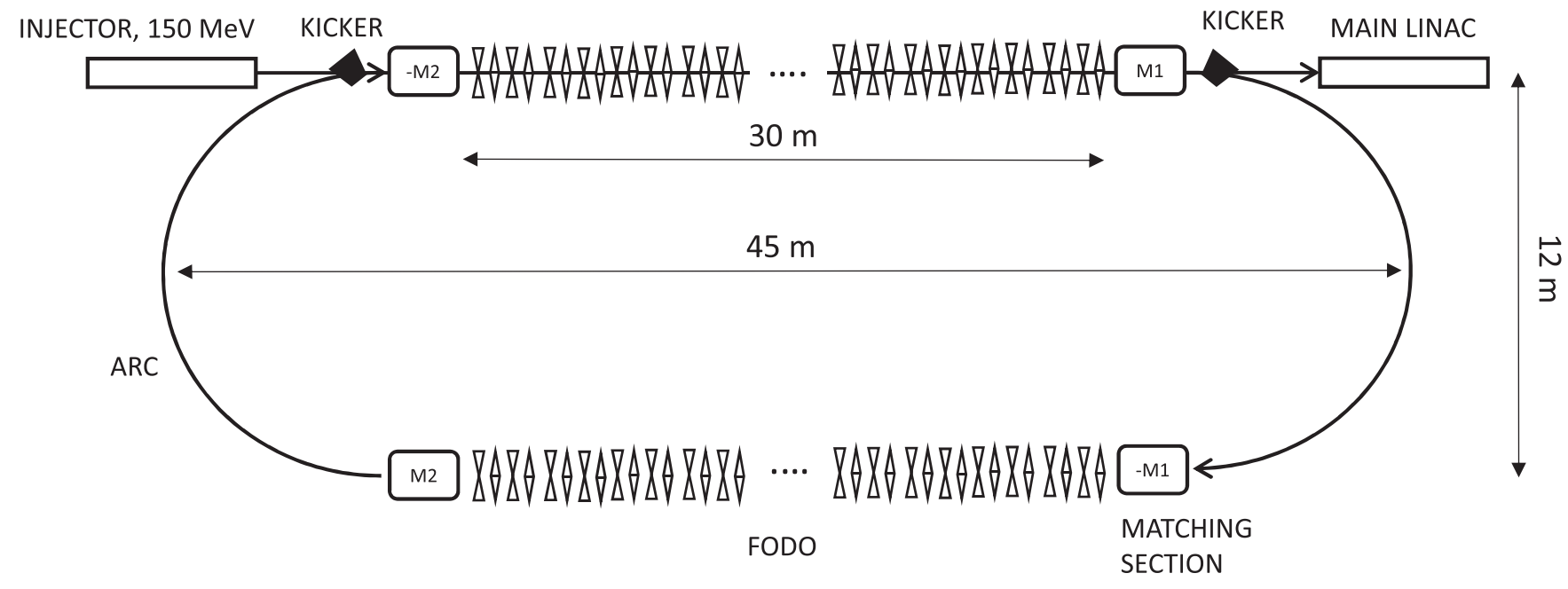

FIG. 5. Schematic layout of the recirculating IBS beam line (not to scale). 



FIG. 6. Electron beam at the entrance of RIBS. Longitudinal phase space (top left), energy distribution (top right) and current profile.

$250 \mathrm{pC}$ beam at the entrance of RIBS. It was generated by including the relativistic velocity spread, the geometric longitudinal wakefield and the rf curvature in an upstream $12 \mathrm{~m}$ long S-band injector. Other beam parameters are: $\varepsilon_{n}=0.4 \mu \mathrm{m} \mathrm{rad}, \sigma_{z}=375 \mu \mathrm{m}$ and $\sigma_{E, 0}=2 \mathrm{keV}$. The total $\mathrm{rms}$ energy spread is $0.1 \%$. This beam is expected to generate $\sigma_{E, \mathrm{IBS}} \approx 10 \mathrm{keV}$ in half a turn [see Eq. (3)], while reducing the strength of the CSR instability with respect to higher beam charges [15].

According to [35] and references therein, a proper balance of the optical functions inside the arc minimizes the CSR-induced emittance growth. At the same time, minimal variation of $R_{56}$ along the arc should weaken the longitudinal CSR instability. The latter prescription was studied in [35] for a high energy arc and is not applied here. For this reason, this RIBS layout illustrates a conservative scenario as far as the longitudinal CSR instability is concerned. The instability is particularly harmful for FELs when the spectral content of the energy modulation extends to wavelengths close to the FEL slippage length [27] $\mathrm{L}_{\mathrm{sl}}=\mathrm{N}_{\mathrm{u}} \lambda$, with $\mathrm{N}_{\mathrm{u}}$ the number of undulator periods. Prior to compression, those modulations of interest are at wavelengths longer by the compression factor C. For typical numbers in $\mathrm{x}$ rays like $\mathrm{N}_{\mathrm{u}} \approx 10^{3}, \lambda=1 \mathrm{~nm}$ and $\mathrm{C}=30$, we find $\mathrm{C} \cdot \mathrm{L}_{\mathrm{sl}} \approx 30 \mu \mathrm{m}$. Thus, the CSR instability may develop in RIBS for wavelengths $\geq 30 \mu \mathrm{m}$. 1.5 million particles were tracked in ELEGANT, with the numerical sampling noise kept under control by following the prescriptions given in [36]. The simulations included CSR in the drift sections following the dipole magnets and transient CSR field at the dipoles' edges [37]. The IBSinduced energy spread along one-turn beam path in RIBS is shown in Fig. 7. The longitudinal phase space is shown in Fig. 8 after a half turn and one turn, respectively. The

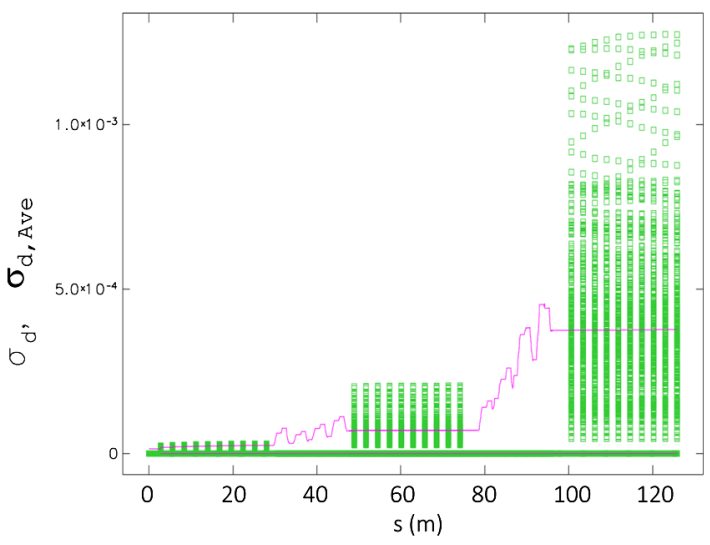

FIG. 7. Slice rms fractional energy spread along RIBS, one turn. Same legend as in Fig. 3. 



FIG. 8. Electron beam longitudinal phase space after a half turn (left) and after one turn in RIBS.
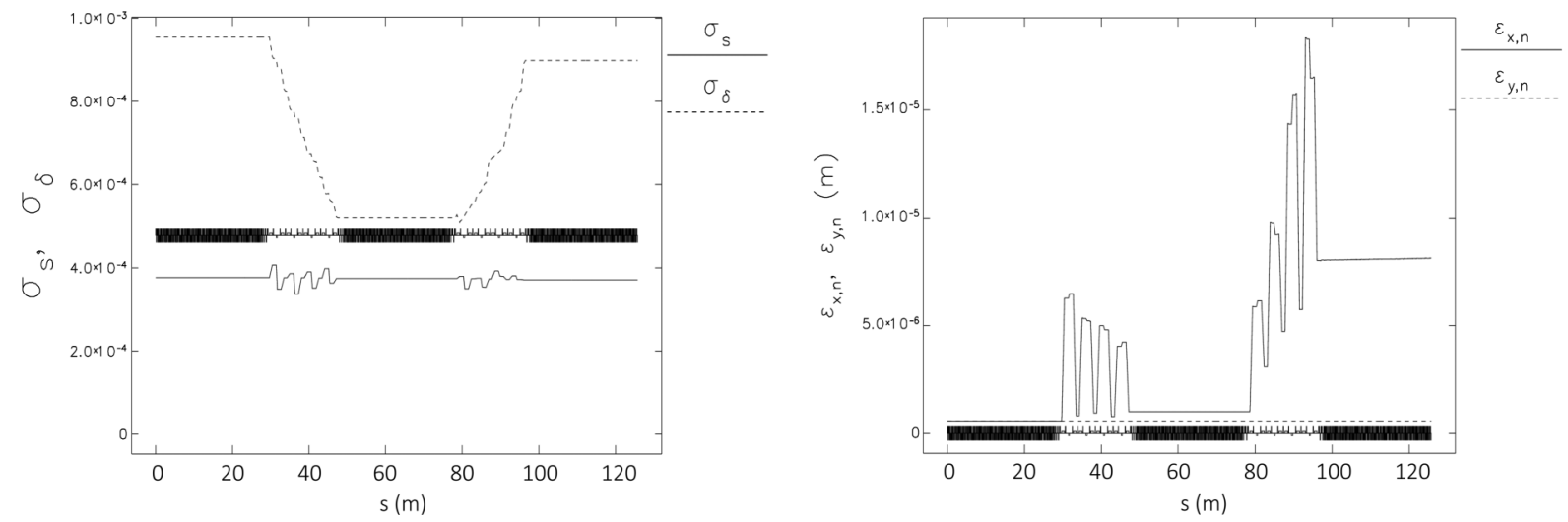

FIG. 9. Left: rms bunch length in meter and total rms fractional energy spread along RIBS, one turn. Right: transverse normalized rms emittances along RIBS, one turn.

evolution of the total energy spread, the bunch length and the transverse normalized projected emittances is shown in Fig. 9.

In principle, the number of turns in RIBS should be a compromise between the amount of desired $\sigma_{\delta \text {,IBS }}$, which is proportional to the square root of the length of the traversed FODO channel, and the tolerable degradation of the beam six-dimensional emittance due to chromatic aberrations and CSR instability. After a half turn, the incoherent energy spread has grown to $10 \mathrm{keV} \mathrm{rms} \mathrm{(see} \mathrm{Fig.} \mathrm{7),} \mathrm{but} \mathrm{largely} \mathrm{at}$ the expense of the deeply modulated longitudinal phase space in Fig. 8. We conclude that the longitudinal CSR instability prevents beam recirculation. In addition, the CSR-induced energy loss modulates the beam correlated energy spread through the arc (see Fig. 9, left plot). This amplifies the variation of the bunch length at the dipole magnets (since $R_{56}$ oscillates in the range $\pm 30 \mathrm{~mm}$ ) and partially invalidates the optics scheme for emittance preservation in the presence of CSR, which requires the same bunch length at the dipoles $[38,39]$. In comparison to the
CSR effect, chromatic aberrations do not play a role as the vertical emittance is preserved all along the beam line (see Fig. 9, right plot).

\section{CONCLUSIONS}

The impact of IBS on the six-dimensional emittance of high brightness electron beams like those driving $\mathrm{x}$-ray FELs has been studied. The analytical estimation based on the Piwinski's formalism is in rough agreement with the particle tracking results obtained with the ELEGANT code. They confirm that IBS is relevant neither to the SASE FEL energy-normalized bandwidth in the ultraviolet-x-ray wavelength range, nor to the gain of the MBI in the main linac.

A low-beta FODO channel has been investigated to increase the longitudinal growth rate of IBS at the linac injection. This solution is far from being as efficient as the LH presently implemented in LCLS and in FERMI: the channel requires tens of quadrupole magnets over tens of meters to generate an incoherent energy spread in the range 
5-10 keV rms, for beam charges in the range 100-500 pC. As an alternative, a recirculating beam line was explored to cumulate IBS-induced energy spread in a relatively compact lattice. Unfortunately, the CSR instability in the arcs, driven by the high charge density and the low beam rigidity, deeply modulates the beam longitudinal phase space even after only a half turn.

In conclusion, a relatively compact single-pass low-beta FODO channel at the linac injection could almost double the incoherent energy spread of high brightness beams with charge in the range $100-500 \mathrm{pC}$. A beam heating above the $10 \mathrm{keV} \mathrm{rms} \mathrm{level} \mathrm{is} \mathrm{envisaged} \mathrm{at} \mathrm{the} \mathrm{end} \mathrm{of} \mathrm{the} \mathrm{FODO}$ channel for charge densities at least $\sim 4$ times higher than generated by state-of-the-art photoinjectors.

\section{ACKNOWLEDGMENTS}

The author thanks M. Cornacchia for suggestions and encouragements. This work was funded by the FERMI project of Elettra-Sincrotrone Trieste, partially supported by the Ministry of University and Research under Grants No. FIRB-RBAP045JF2 and No. FIRB-RBAP06AWK3.

[1] Z. Huang, Technical Note No. LCLS-TN-02-8, 2002.

[2] A. Xiao and M. Borland, in Proceedings of the 23rd Particle Accelerator Conference, Vancouver, Canada, 2009 (IEEE, Piscataway, NJ, 2009), TH5PFP038.

[3] M. Borland, APS Report No. LS-287, 2000.

[4] A. Xiao, in Proceedings of the 24th Linear Accelerator Conference, Victoria, BC, Canada (2008), MOP093.

[5] A. Piwinski, in Intrabeam Scattering, edited by S. Turner, CERN Accelerator School: 4th General Accelerator Physics Course (CERN, Jülich, Germany, 1991), p. 226.

[6] A. Piwinski, in Proceedings of the 1983 SSC Workshop, Ann Arbor, MI (1983), p. 59.

[7] K. Kubo and K. Oide, Phys. Rev. ST Accel. Beams 4, 124401 (2001).

[8] S. Nagaitsev, Phys. Rev. ST Accel. Beams 8, 064403 (2005).

[9] K. Kubo, S. K. Mtingwa, and A. Wolski, Phys. Rev. ST Accel. Beams 8, 081001 (2005).

[10] Z. Huang, M. Borland, P. Emma, J. Wu, C. Limborg, G. Stupakov, and J. Welch, Phys. Rev. ST Accel. Beams 7, 074401 (2004).

[11] S. Spampinati et al., in Proceedings of the 34th Free Electron Laser Conference, Nara, Japan (2012), WEPPH015.

[12] P. Emma et al., Nat. Photonics 4, 641 (2010).
[13] E. Allaria et al., Nat. Photonics 6, 699 (2012).

[14] E. L. Saldin, E. A. Schneidmiller, and M. V. Yurkov, Nucl. Instrum. Methods Phys. Res., Sect. A 490, 1 (2002).

[15] Z. Huang and K. J. Kim, Phys. Rev. ST Accel. Beams 5, 074401 (2002).

[16] S. Heifets, G. Stupakov, and S. Krinsky, Phys. Rev. ST Accel. Beams 5, 064401 (2002).

[17] M. Hüning and H. Schlarb, in Proceedings of the 2003 Particle Accelerator Conference, Portland, OR (IEEE, New York, 2003), WUPAB017.

[18] E. L. Saldin, E. A. Schneidmiller, and M. V. Yurkov, Nucl. Instrum. Methods Phys. Res., Sect. A 528, 355 (2004).

[19] C. Behrens, Z. Huang, and D. Xiang, Phys. Rev. ST Accel. Beams 15, 022802 (2012).

[20] J. Qiang, C. E. Mitchell, and M. Venturini, Phys. Rev. Lett. 111, 054801 (2013).

[21] S. Di Mitri and S. Spampinati, Phys. Rev. Lett. 112, 134802 (2014).

[22] Z. Huang et al., Phys. Rev. ST Accel. Beams 13, 020703 (2010).

[23] S. C. Leemann, Phys. Rev. ST Accel. Beams 17, 050705 (2014).

[24] M. P. Ehrlichman, W. Hartung, B. Heltsley, D. P. Peterson, N. Rider, D. Rubin, D. Sagan, J. Shanks, S. T. Wang, R. Campbell, and R. Holtzapple, Phys. Rev. ST Accel. Beams 16, 104401 (2013).

[25] K. L. F. Bane, H. Hayano, K. Kubo, T. Naito, T. Okugi, and J. Urakawa, Phys. Rev. ST Accel. Beams 5, 084403 (2002).

[26] T. Raubenheimer, Part. Accel. 45, 111 (1994).

[27] R. Bonifacio, C. Pellegrini, and L. Narducci, Opt. Commun. 50, 373 (1984).

[28] A. M. Kondratenko and E. L. Saldin, Part. Accel. 10, 207 (1980).

[29] K.-J. Kim, Phys. Rev. Lett. 57, 1871 (1986).

[30] G. De Ninno and E. Allaria, Phys. Rev. Lett. 99, 014801 (2007).

[31] G. Stupakov, Phys. Rev. Lett. 102, 074801 (2009).

[32] G. R. White et al., Phys. Rev. Lett. 112, 034802 (2014).

[33] For a summary of the experimental data we refer to S. Di Mitri, Phys. Rev. ST Accel. Beams 16, 050701 (2013), and references therein.

[34] J. D. Bjorken and S. K. Mtingwa, Part. Accel. 13, 118 (1983).

[35] D. R. Douglas et al., arXiv:1403.2318.

[36] M. Borland, Phys. Rev. ST Accel. Beams 4, 070701 (2001).

[37] G. Stupakov and P. Emma, SLAC Report No. LCLS-TN01-12, 2001.

[38] D. Douglas, Report No. JLAB-TN-98-012, 1998.

[39] S. Di Mitri, M. Cornacchia, and S. Spampinati, Phys. Rev. Lett. 110, 014801 (2013). 